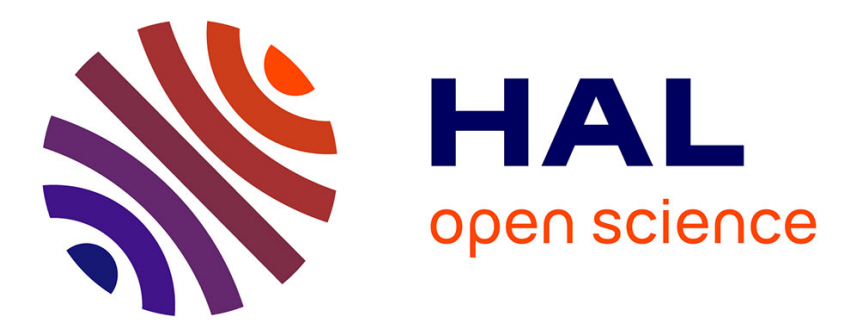

\title{
Radio wave propagation in arch-shaped tunnels: Measurements and simulations by asymptotic methods
}

Emilie Masson, Pierre Combeau, Yann Cocheril, Marion Berbineau, Lilian Aveneau, Rodolphe Vauzelle

\section{- To cite this version:}

Emilie Masson, Pierre Combeau, Yann Cocheril, Marion Berbineau, Lilian Aveneau, et al.. Radio wave propagation in arch-shaped tunnels: Measurements and simulations by asymptotic methods. Comptes Rendus de l'Academie des Sciences. Série IV, Physique, Astronomie, 2010, 11 (1), pp.44 53. 10.1016/j.crhy.2009.12.003 . hal-00487167

\section{HAL Id: hal-00487167 https://hal.science/hal-00487167}

Submitted on 26 Oct 2012

HAL is a multi-disciplinary open access archive for the deposit and dissemination of scientific research documents, whether they are published or not. The documents may come from teaching and research institutions in France or abroad, or from public or private research centers.
L'archive ouverte pluridisciplinaire HAL, est destinée au dépôt et à la diffusion de documents scientifiques de niveau recherche, publiés ou non, émanant des établissements d'enseignement et de recherche français ou étrangers, des laboratoires publics ou privés. 


\title{
Propagation des ondes radioélectriques en tunnels voûtés : mesures et simulations par méthodes asymptotiques
}

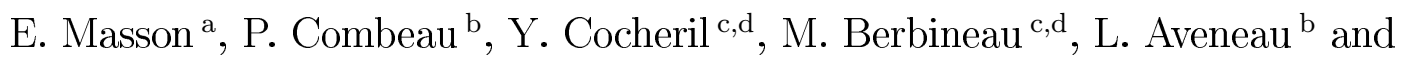 \\ R. Vauzelle ${ }^{\mathrm{b}}$ \\ ${ }^{\mathrm{a}}$ A LSTOM-Transport, 33 rue des bateliers, F-93400 Saint-Ouen, France \\ ${ }^{\mathrm{b}}$ Univ Poitiers Xlim-SIC CNRS UMR 6172, Bd Curie, F-86962 Futuroscope-Chasseneuil, France \\ ${ }^{\mathrm{c}}$ Univ Lille Nord de France, F-59000 Lille, France \\ ${ }^{\mathrm{d}}$ INRETS, LEOST, 20 rue Elisée Reclus, F-59650 Villeneuve d'Ascq, France
}

Reçu le 1er septembre 2009 ; accepté après révision le +++++

\begin{abstract}
Résumé
De nombreux systèmes de communication sans fil sont développés pour les communications entre les trains et l'infrastructure et entre les trains dans les domaines métro et ferroviaire. Ils répondent à des besoins opérationnels de sécurité et de confort. Dans le but de déployer ces systèmes dans les environnements particuliers que sont les tunnels, qui peuvent être rectilignes ou courbes, et de section rectangulaire ou voûtée, des modèles de propagation spécifiques doivent être développés. Une modélisation de la propagation en tunnels rectilignes de section voûtée a été réalisée à l'aide de différents modèles asymptotiques à base de tracé et de lancer de rayons, alliés à une facettisation de la section voûtée. Une méthode d'interpolation des normales a été implémentée afin de minimiser l'erreur introduite par l'utilisation de la facettisation. Les résultats obtenus ont été validés par confrontation à la littérature et à des résultats de mesures effectuées dans un tunnel de section voûtée.
\end{abstract}

\begin{abstract}
Radio Wave Propagation in Arch-Shaped Tunnels : Measurements and Simulations using Asymptotic Methods. Several wireless communication systems are developed for communication needs between train and ground and between trains in the railway or mass transit domains. They are developed for operational needs for security and comfort. In order to deploy these systems in specific environments, such as tunnels, straight or curved, rectangular or arch-shaped section, specific propagation models have to be developed. A modelisation of the radio wave propagation in straight arch-shaped tunnels is realized by using asymptotic methods, such as Ray Tracing and Ray Launching, combined with the tessellation of the arched section. A method of interpolation of the facets' normals was implemented in order to minimize the error made when using the tessellation. Results obtained are validated by comparison to the literature and to measurement results.
\end{abstract}

Key words: Radio Wave Propagation; Tunnels; Asymptotic Methods; Measurements

Mots-clés : Propagation des ondes radio ; Tunnels ; Méthodes asymptotiques ; Mesures

Email addresses: emilie.masson@inrets.fr (E. Masson), combeau@sic.sp2mi.univ-poitiers.fr (P. Combeau), yann.cocheril@inrets.fr (Y. Cocheril), marion.berbineau@inrets.fr (M. Berbineau), aveneau@sic.sp2mi.univ-poitiers.fr (L. Aveneau), vauzelle@sic.sp2mi.univ-poitiers.fr (R. Vauzelle). 


\section{Introduction}

Wireless communication systems are developed in the guided transport domain (metro, classical trains, High Speed Trains (HST)) for communications between train and infrastructure and between trains. These systems answer to operational needs for security and comfort, such as traffic management, maintenance or security of passengers and staff. Among these applications, we can mention control-command systems, high data-rate transmissions for video surveillance or entertainment. The prediction of radio coverage levels is required to optimize systems deployment and ensure availability and robustness of the radio links. Generally, minimal field levels are required to guarantee key performance indicators related to safety constraints or Quality of Service (QoS). These systems operate in complex radio environments, such as tunnels. In these last ones, the usual laws of free space propagation are no longer valid and the propagation phenomenon has to be specifically analyzed. Thus, up to now, the case of tunnel areas is generally treated via intensive measurement campaigns. The development of specific propagation models is then very relevant. Realistic tunnels can be of rectangular cross section or arch-shaped, and straight or curved.

In order to describe the radio wave propagation in tunnels, several modeling approaches can be set up. The numerical resolution of Maxwell's equations would be an ideal solution. However, this kind of techniques is not feasible due to the huge computational burden. A more conventional way to solve the problem is given by modal theory. The tunnel is here treated as a hollow waveguide with dielectric boundaries. Unfortunately, analytical expressions of the different constants, such as cut-off frequency and wave impedance, only exist for few canonical types of configuration, e.g. rectangular or circular cross section waveguides [Bal89]. The prediction of radio wave propagation with an adequate accuracy in finite time is given by ray optics solutions. These solutions can be adopted in tunnels because the dimensions are generally large compared to the considered wavelength (frequency above $1 \mathrm{GHz}$ ). Several methods based on the ray-optical modeling approach have been proposed. They use Ray Launching [ZHK98], [WY06], Ray Tracing [TVD ${ }^{+} 99$ ] or combination of each [CJ96]. The classical approach of Ray Tracing techniques cannot be transposed to the case of curved surfaces. Indeed, the image method is not applicable because of the infinite line of images of one source compared to a single point for planar surfaces. For the Ray Launching, the concept of reception sphere is no longer valid for curved surfaces because of the nonconservation of reflection angles [Did00]. The first conceivable solution is the development of a completely novel model based on ray optics, as in [Did00], [DMW01]. The second option is to consider the equivalent rectangular cross section tunnel with an equal area [MGPLND08]. We have chosen to tessellate geometries into multiple planar facets, as proposed in [CJ96], [TVD ${ }^{+}$99], [BBD98].

In this paper, radio wave propagation in straight arch-shaped tunnels is analyzed by using asymptotic methods combined with tessellation in multiple planar facets of the arched cross section, in order to approximate the curvature. Simulations were realized at $1 \mathrm{GHz}$ in order to compare with literature results. Three methods are analyzed and compared. Section 2 presents the tessellation method combined with a Ray Tracing technique, based on image method. Results and limits of this method in proposed configurations are presented. In Section 3, Ray Launching is implemented and tested. An algorithm of Identification of Multiple Rays (IMR) is implemented and added to the Ray Launching. As a first step, the geometric facets' normals are used to compute the reflection of rays on facets. In order to eliminate errors introduced by the tessellation, a method of interpolation of the facets' normals is then implemented, similar to the Phong algorithm [Gla94]. In Section 4, some measurement results at $5.8 \mathrm{GHz}$ in an arch-shaped tunnel are presented and compared with simulations at the same frequency. Finally, conclusions and perspectives are given in Section 5.

\section{Tessellation combined with Ray Tracing}

Ray Tracing [SR94] is an asymptotic method that consists of a direct search for geometric paths followed by the waves. It allows us to exactly determine the set of paths from a transmitter to a receiver. This technique relies on the image theory based on Snell-Descartes formulas. The set of images of the transmitter is computed in relation to the environment's facets, for a given number of successive reflections. From these paths traced according to Snell-Descartes laws, the electric field is computed from Geometrical Optic (GO) laws and Fresnel coefficients. All the rays are combined in a coherent way at receiver to compute the power at the punctual position of the receiver, after having taken into account the depolarization of waves after reflections. For all our simulations, the maximum authorized number of reflections is 10 . 
In the case of a curved surface, one source generates an infinite number of images. To solve this problem, we chose to approximate the curved surface by a moderate number of facets, in order to keep a reasonable computation time.

In order to compare to literature results, simulations were realized at $1 \mathrm{GHz}$ in a straight arch-shaped tunnel, in the configuration illustrated in Figure 1. The transmitter is located in the center of the arch, at a height of $4.5 \mathrm{~m}$. The receiver is set up $2 \mathrm{~m}$ from the sidewall, at a height of $1.5 \mathrm{~m}$ and moves along the tunnel axis. This configuration was chosen to avoid symmetrical effects [CJ96].

Figure 2 (a) illustrates the results obtained in terms of received power $\left(P_{r}\right)$ in comparison to transmitted power $\left(P_{t}\right)$ versus the distance between transmitter and receiver along the longitudinal tunnel axis, depending on the number of facets used to approximate the arch-shaped cross section. The number of facets has a significant effect on the received power. We propose in the following to analyze these results by studying signals statistic of the difference between the results for $n$ and $n+1$ facets. This method allow us to analyze the convergence or no convergence of results and to compare the speed of convergence between the different simulation techniques described in this paper. First of all, we smooth the signals by using a running mean. The window's length is $40 \lambda$, on the first 50 meters, and $100 \lambda$ for the rest [LD98]. Then, the mean and standard deviation (in $\mathrm{dB}$ ) of the difference between the results for $n$ and $n+1$ facets depending on the number of facets are computed in order to analyze the behavior while increasing the number of facets. Figures 2 (b) and (c) highlight an unpredictable behavior of the signals by increasing the number of facets. It appears that for a reasonable number of facets, received power does not converge. The results are different from Wang and Yang [WY06]. This can be explained by the fact that, for each additional facet, new paths are computed but do not correspond to real ones. The received power is then badly estimated, it is overvalued. This phenomenon would increase by using more facets. Furthermore, it causes an exponential increase of the computation time, which becomes impractical.

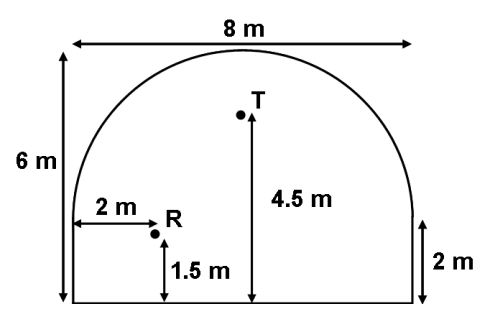

Figure 1. Configuration of simulation


(a) Simulated path loss versus the dis- (b) Mean between results for $n$ and $n+1$ (c) Standard deviation between results tance depending on the number of facets facets versus the number of facets for $n$ and $n+1$ facets versus the number of facets

Figure 2. Ray Tracing results

\section{Tessellation combined with Ray Launching}

Section 2 highlights a non convergence of the simulated power and the difficulty to increase the number of facets because of the exponential increase of computation time. In this part, we implement a Ray Launching method in order to avoid this phenomenon and then we analyze the results. 


\subsection{Use of Facets' normals}

Ray Launching [SR94] is an asymptotic method that consists in launching a large number of rays from the transmitter into the environment. It consists to sample the infinite set of rays radiated by the transmitter, using rays starting from the transmitter position and then propagating in the scene. For sampling the radiated rays, we use a Quasi Monte-Carlo technique [MCN]. It is very similar to Monte Carlo techniques [Ham64], but in in this case, random numbers are chosen to regularly cover all the space. Many low discrepancy sequences exist, we chose to use simple ones, the Hammersley sequences [MCN].

The rays reaching the receiver have to be determined. Thereby, a sphere is considered around the receiver and each ray intercepting this sphere is considered as contributing to the received power. Thus, several geometric similar rays can be retained, even though one single path exists effectively for the Geometrical Optic meaning. The estimation of the received power is then distorted (such as for the Ray Tracing case in Section 2). It represents the multiple rays phenomenon. Thus, the difficulty of this method lies in the adjustment of the sphere size around the receiver and the criteria of the Identification of Multiple Rays (IMR) [IY02]. The retained IMR algorithm consists in evaluating the following criteria :

(i) number of reflections : if the number of reflections of two rays is equal, then we evaluate the second criterion,

(ii) ray length : the lengths (or delay) of the two rays are compared. If both fall into the same length interval, they are defined equal, and we evaluate the last criterion,

(iii) direction of Departure (DoD) : the DoD of the two rays are compared. If both are launched in the same direction, the rays are considered to be multiple and we keep only one of the two rays.

If the first criterion is unambiguous, the following two require the specification of a given test range, which has to be fixed adequately. After tests performed with the simulator, the interval of ray length was fixed at 1 meter. For the third criterion, the angle between the two rays is compared, the interval was fixed at 2.5 degrees.

The Figure 3 (a) illustrates the results obtained by using Ray Launching combined with the IMR algorithm in terms of received power $\left(P_{r}\right)$ in comparison to transmitted power $\left(P_{t}\right)$ versus the distance between transmitter and receiver along the longitudinal tunnel axis, depending on the number of facets. The use of Ray Launching techniques allows us to increase the number of facets (here 200 facets). A convergence of results to a same signal level seems to be observed. To prove this, we applied the same procedure than in Section 2. The results are illustrated in Figures 3 (b) and (c). The mean tends to 0 and the standard deviation decreases with the number of facets : it highlights a convergence of the results by increasing the number of facets. Furthermore, results are converging to the same results than Wang and Yang [WY06].


(a) Simulated path loss versus the dis- (b) Mean between results for $n$ and $n+1$ (c) Standard deviation between results tance depending on the number of facets facets versus the number of facets for $n$ and $n+1$ facets versus the number of facets

Figure 3. Ray Launching results

\subsection{Normals' interpolation}

The tessellation of the arched section leads to an error on the normals at reflection points. Indeed, whatever the reflection point is, the corresponding normal is systematically the facet's normal, as shown in Figure 4. This example shows that if the facet's normal is considered, the path is not received, while, in reality, using the exact 
normal of the curved surface, the path would have been intercepted at reception. In the same time, by using the facets, other wrong paths are received.

We present here a method allowing us to minimize this error by estimating the normal of the curved surface at the reflection point. This method consists in a linear interpolation of the facets' normals, like in the Phong algorithm [Gla94], widely used in image synthesis domain. The interpolation is based on barycentric coordinates in a triangle of the reflection point $P$. The principle is based on a pre-computation, for each facet, of the normal of each of the four vertices of the facet. Each vertex normal is fixed as the mean of adjoining facets' normals, if the angle between facets is not too large. Then, for any reflection point $P$ on a given facet $F$, we linearly interpolate the normals of $F$ 's vertices, giving a good estimation of the normal at $P$.

Figures 5 (a) and (b) present the impact of interpolation in the transverse section. The results in Ray Launching (with reception sphere and IMR) obtained in the previous tunnel approximated by 3 and 200 facets are presented. The receiver moves along the cross section from $-3.5 \mathrm{~m}$ to $3.5 \mathrm{~m}$ from the middle of the tunnel, 5 meters from the transmitter. Figure 5 (a) shows a large difference between results obtained with and without interpolation in the 3 facets tunnel. To highlight this observation, we present in Figure 6 (a) and (b) the power level distributions when the transmitter is located at a $2.5 \mathrm{~m}$ horizontal plan in the 3 facets tunnel with and without interpolation. Important differences clearly appear between the two configurations. Discontinuities of signal level are observed for the configuration without interpolation. Figure 5 (b) compares the results obtained with 3 facets and 200 facets in the transverse section, using interpolation. We can see that results are very closed. One more time, the comparison of the power level distributions for a 3 and 200 facets tunnel with interpolation presented in Figure 6 (b) and (c) confirm this observation. The interpolation technique allows us to eliminate the discontinuities in the power level distribution, caused by the tessellation.

The Figure 7 (a) illustrates the results obtained in terms of received power $\left(P_{r}\right)$ in comparison to transmitted power $\left(P_{t}\right)$ versus the distance between transmitter and receiver along the longitudinal tunnel axis, depending on the number of facets, by using Ray Launching combined with the IMR algorithm and the interpolation of facets' normals. Once again, we applied the procedure of Section 2 to analyze the convergence of results. It is illustrated in Figures 7 (b) and (c). The mean tends more rapidly to 0 while the standard deviation decreases rapidly. A distinct and fast convergence is observed on the signal levels by using Ray Launching techniques combined with the interpolation, while increasing the number of facets. The results are also very close to Wang and Yang results [WY06] using another technique. This can be a first validation of our approach.

The three propagation simulation method were analyzed and compared. The received power seems to be badly estimated using Ray Tracing and the computation time increase exponentially with the increase of the facet's number. The Ray Launching technique avoids this phenomenon. A convergence of signals to a same power level is observed. The interpolation method speeds up this convergence. The following section presents some measurement results used as a reference to compare and validate the simulation techniques.

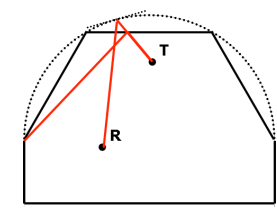

Figure 4. Illustration of the error induced on the normals
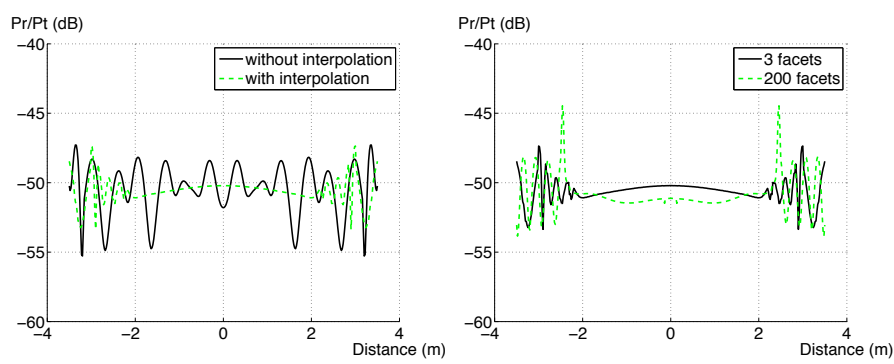

(a) In a 3 facets tunnel without-with (b) In a 3 and 200 facets with interinterpolation polation

Figure 5. Impact of interpolation in the transverse section 


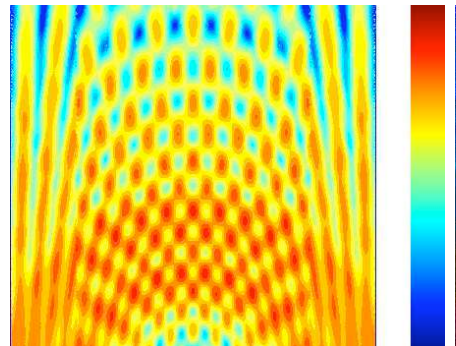

(a) 3 facets without interpolation

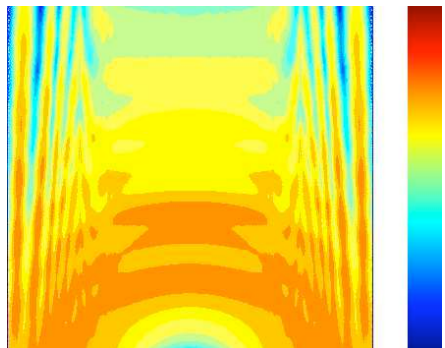

(b) 3 facets with interpolation

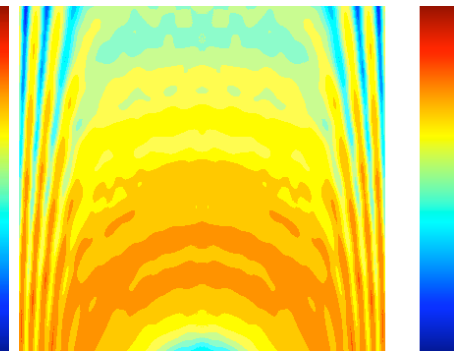

(c) 200 facets with interpolation

Figure 6. Power level distributions $(8 \mathrm{mx} 8 \mathrm{~m})$ at a $2.5 \mathrm{~m}$ horizontal plane

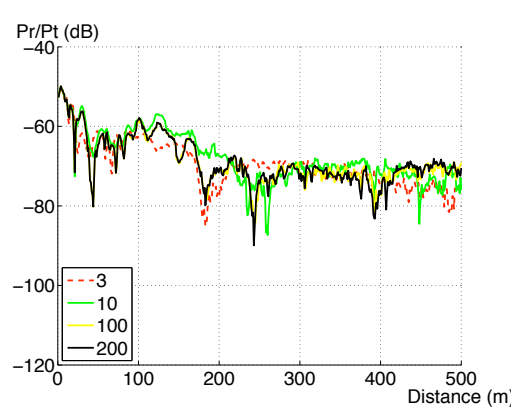

(a) Simulated path loss versus the dis- (b) Mean between results for $n$ and $n+1$ tance depending on the number of facets facets versus the number of facets

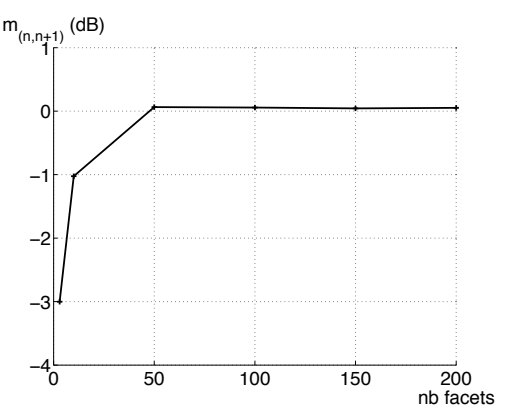



(c) Standard deviation between results for $n$ and $n+1$ facets versus the number of facets

FIgure 7. Ray Launching combined with interpolation results

\section{Measurement results}

\subsection{Trial conditions}

Measurements were conducted in the Tunnel of Roux, which is a two-way straight arch-shaped road tunnel located in the Ardèche region of France. This tunnel is perfectly straight and has a length of $3.336 \mathrm{~km}$. The transverse section of the tunnel is semicircular and has a diameter of $8.3 \mathrm{~m}$. The maximum height is $5.8 \mathrm{~m}$ at the center of the tunnel. Measurements were conducted at $5.8 \mathrm{GHz}$. The transmitting part is static. It is composed of a large bandwidth horn antenna, $10.1 \mathrm{dBi}$ gain at $5.8 \mathrm{GHz}$, vertically polarized, and connected by a low loss cable, $100 \mathrm{~dB} / 100 \mathrm{~m}$ at $5.8 \mathrm{GHz}$, to a transmitter delivering a sinusoidal signal at the required frequency. The mobile reception system is composed of the same horn antenna, also vertically polarized, and connected by a low loss cable to a THALES VUH-TRC8025 receiver used in an analyzer mode. The system is fixed up on a go-kart allowing a very small and regular velocity, compatible with the acquisition rate.

Figure 8 illustrates the measurement configuration. The transmitter is located almost at the center of the section, at a height of $4.8 \mathrm{~m}$. The moving receiver is located in the middle of one of the two tracks, $2.4 \mathrm{~m}$ from the sidewall, at a height of $4.1 \mathrm{~m}$.

\subsection{Simulation and measurement comparison}

All the results presented are normalized by the maximum of the received power along the tunnel in order to cancel the errors that could be introduced by antenna gain and cable loss. Figures 9 (a), (b) and (c) illustrate the measurement results compared to simulation results obtained at $5.8 \mathrm{GHz}$ using tessellation of the cross section, in the same configuration. Figure 9 (a) shows the comparison between measurements and simulations using Ray Tracing. Figure 9 (b) and (c) illustrate the comparison between measurements and simulations using Ray Launching, respectively without and with the technique of interpolation. We choose to take into account 50 facets for the approximation of the arched section in order to keep reasonable computation time in Ray Tracing. 
The over evaluation of signal level in Ray Tracing and the good concordance of Ray Launching are confirmed by the results observed in those figures. In the following, we analyze these results by studying the statistics of slow and fast fading.



Figure 8. Tunnel of Roux

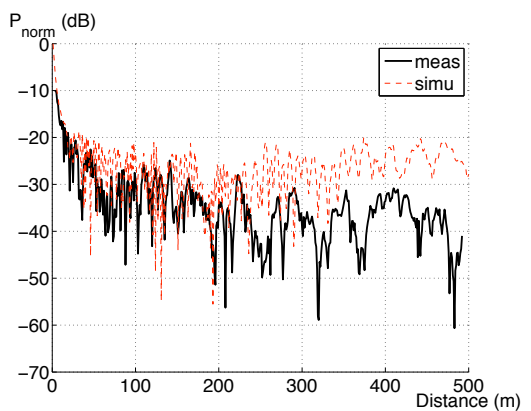

(a) Ray Tracing

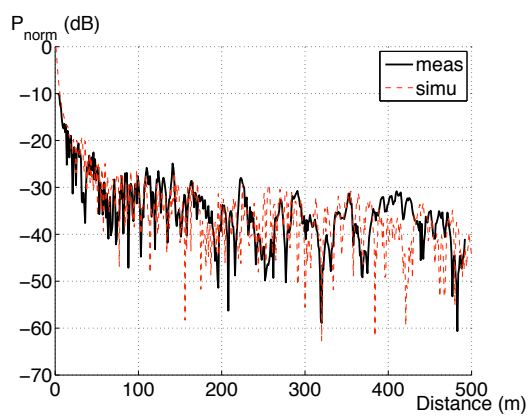

(b) Ray Launching without interpolation

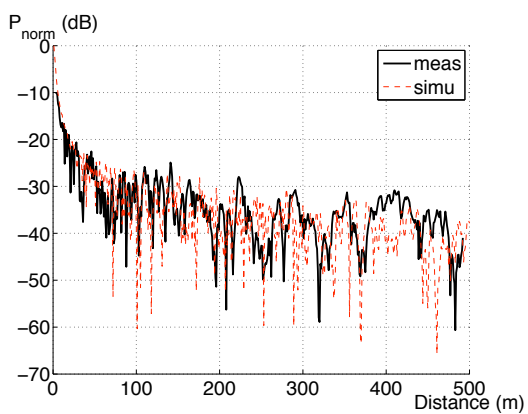

(c) Ray Launching with interpolation

Figure 9. Measured and Simulated path loss using 50 facets as a function of the distance

\subsubsection{Slow fading statistics}

To subtract fast fading variations, we smooth the received signal by the same procedure than in previous sections. Then, the mean and standard deviation of the error between the measured and simulated mean powers are computed. Results obtained are illustrated in Table 1. The results in terms of mean highlight a bad estimation of signal levels in Ray Tracing and a good agreement between measurement and simulation results while using Ray Launching. This confirms what was observed in Section 2. No specific analyze can be done on standard deviations obtained; a similar behavior in Ray Tracing and Ray Launching is observed.

TABLE 1

Mean and standard deviation between Measurements and Simulations (50 facets)

\begin{tabular}{|c|c|c|}
\hline Simulation method & Mean (dB) & Standard deviation (dB) \\
\hline \hline Ray Tracing & 8.58 & 5.93 \\
\hline Ray Launching without interpolation & 1.13 & 5.05 \\
\hline Ray Launching with interpolation & 0.18 & 4.93 \\
\hline
\end{tabular}

\subsubsection{Fast fading statistics}

The previous section allowed us to evaluate the three methods. Ray Tracing combined with tessellation gives a bad estimation of the received power and the computation time increases very rapidly. Ray Launching avoids this phenomenon and leads up to a convergence of results. The addition of the interpolation of facet's normals gives 
better results. The signal levels converge quite well to the measurement results. In this part, we realize a analyze of fast fading on results given by Ray Launching combined with the interpolation, for a tessellation on 200 facets.

The statistical analysis of fast fading is realized on the measurement and simulation results. The two previous windows (the first of length $40 \lambda$ on the first 50 meters, the second equal to $100 \lambda$ for the rest) are used to extract the fast variations. The Cumulative Density Functions (CDF) of the measured and simulated data are calculated. They are both compared to the Rayleigh, Nakagami and Weibull distributions. We used the Kolmogorov-Smirnov test in order to decide which distribution best fits the results. Figure 10 shows the CDF of the simulated and measured data compared to the models. The parameters of the theoretical distributions are determined by Maximum Likelihood estimators. Table 2 contains the Kolmogorov-Smirnov (KS) criteria of the different distributions and the estimated values of the parameters of the statistics, respectively. Figure 10 highlights a good matching with the Weibull distribution for simulation and measurement results. Table 2 confirms this observation. The distribution which minimizes the Kolmogorov-Smirnov (KS) criterion is the Weibull distribution. The results highlight a similar behavior in terms of fast fading statistics for measurements and simulations.

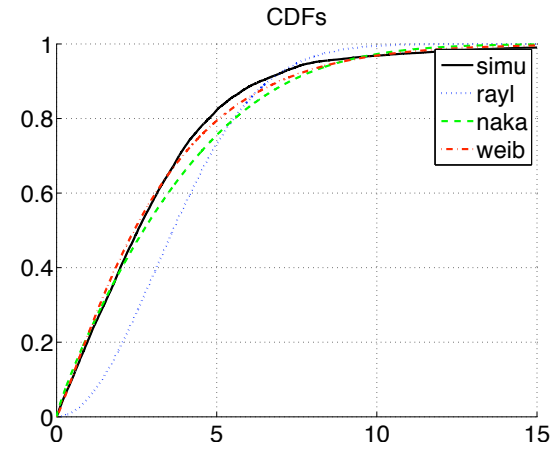

(a) Simulated data compared to fitted distributions

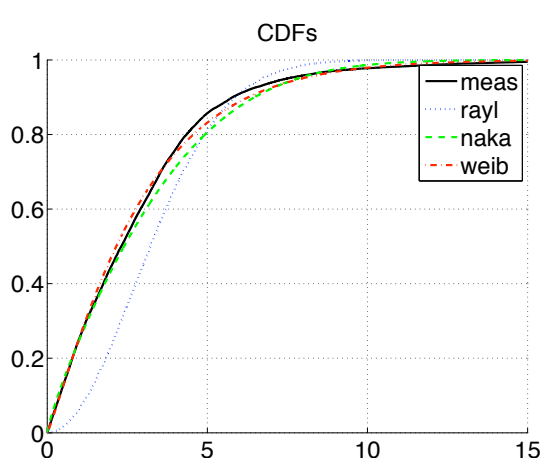

(b) Measured data compared to fitted distributions

Figure 10. Measured and Simulated path loss using 200 facets as a function of the distance

TABLE 2

KS criteria between signal and fitted distribution and values of fitted statistic distributions

\begin{tabular}{|c|c|c|c|}
\cline { 3 - 4 } \multicolumn{2}{c|}{} & Measurement & Simulation \\
\hline \hline \multirow{4}{*}{ Rayleigh } & $\mathrm{KS}$ & 0.21 & 0.21 \\
\cline { 2 - 4 } & $\sigma$ & 2.74 & 3.08 \\
\hline \multirow{3}{*}{ Weibull } & $\mathrm{KS}$ & 0.05 & 0.07 \\
\cline { 2 - 4 } & $m$ & 0.42 & 0.42 \\
\cline { 2 - 4 } & $\omega$ & 14.98 & 19.04 \\
\cline { 2 - 4 } & $\mathrm{KS}$ & 0.03 & 0.03 \\
\cline { 2 - 4 } & $l$ & 2.99 & 3.34 \\
\hline
\end{tabular}

\section{Conclusion}

This paper presented three methods to model the radio wave propagation in straight arch-shaped tunnels. A tessellation of the section was used combined with asymptotic methods. First, a Ray Tracing tool was tested. This technique shows its limits in such tunnel geometry. The results do not converge to the literature results. Indeed, for each facet added, new paths are added and the received power is badly estimated. Moreover, the Ray Tracing technique is limited by the number of facets used to approximate the arch. An exponential increase of computation time is observed by increasing this number, which becomes prohibitive. We propose a solution to avoid this problem by implementing a Ray Launching method. The difficulty of this method lies in the adjustment 
of the reception sphere and the criteria of the Identification of Multiple Rays (IMR). An interpolation of the facets' normals is implemented in order to minimize the error made on normals by using tessellation. This method allows us to observe a convergence of the results by considering a large number of facets. Finally, measurements in a straight arch-shaped tunnel were realized at the $5.8 \mathrm{GHz}$ frequency. A good concordance between simulations and measurements and a similar behavior in terms of fast fading is observed. The results obtained in Ray Launching with the interpolation of the facets' normals converge quite well to the measurement results. We envisage, as the continuation of this work, to compare the results with those obtained with exact methods, such as FDTD.

\section{Acknowledgments}

The authors would like to thank the ALSTOM-TIS (Transport Information Solution) who supported this work.

\section{Références}

[Bal89] C. A. Balanis. Advanced Engineering Electromagnetics. John Wiley and Sons, 1989.

[BBD98] S. Baranowski, G. Bourdier, and P. Degauque. Optimisation des règles d'ingénierie radio - modélisation de la propagation d'ondes radioélectriques en tunnels courbes. Convention d'études INRETS/USTL 1997/L1, Laboratoire de Radiopropagation et Electronique, Villeneuve d'Ascq, avril 1998.

[CJ96] S.-H. Cheng and S.-K. Jeng. SBR image approach for radio wave propagation in tunnels with and without traffic. IEEE Transactions on Antennas and Propagation, 45(3) :570-578, 1996.

[Did00] D. Didascalou. Ray-optical Wave Propagation Modelling in Arbitrarily Shaped Tunnels. Ph.D. dissertation, University of Karlsruhe, Germany, fév 2000.

[DMW01] D. Didascalou, J. Maurer, and W. Wiesbeck. Subway tunnel guided electromagnetic wave propagation at mobile communications frequencies. IEEE Transactions on Antennas and Propagation, 49(11):1590-1596, 2001.

[Gla94] A. S. Glassner. Principles of Digital Image Synthesis. ISBN 1558602763. Morgan Kaufmann Publishers Inc., 1994.

[Ham64] J. M. Hammersley. Monte Carlo methods. 1964.

[IY02] M. F. Iskander and Z. Yun. Propagation prediction models for wireless communication systems. IEEE Transaction on Microwave Theory and Techniques, 50(3) :662-673, 2002.

[LD98] M. Lienard and P. Degauque. Propagation in wide tunnels at $2 \mathrm{GHz}$ : A statistical analysis. IEEE Transactions on Vehicular Technology, 47(4) :1322-1328, 1998.

[MCN] W. J. Morokoff, R. E. Caflisch, and O. Numbers. Quasi-monte carlo integration.

[MGPLND08] J. M. Molina-García-Pardo, M. Lienard, A. Nasr, and P. Degauque. On the possibility of interpreting field variations and polarization in arched tunnels using a model for propagation in rectangular or circular tunnels. IEEE Transactions on Antennas and Propagation, 56(4) :1206-1211, 2008.

[SR94] S.Y. Seidl and T.S. Rappaport. Site-specific propagation prediction for wireless in-building personal communication system design. IEEE Transactions on Vehicular Technology, 43(3) :879-891, 1994.

$\left[\mathrm{TVD}^{+} 99\right] \quad$ R. P. Torres, L. Valle, M. Domingo, S. Loredo, and M. C. Diez. CINDOOR : An engineering tool for planning and design of wireless systems in enclosed spaces. IEEE Antennas and Propagation Magazine, 41(4), 1999.

[WY06] T.-S. Wang and C.-F. Yang. Simulations and measurements of wave propagations in curved road tunnels for signals from GSM base stations. IEEE Transactions on Antennas and Propagation, 54(9) :2577-2584, 2006.

[ZHK98] Y.P. Zhang, Y. Hwang, and R.G. Kouyoumjian. Ray-optical prediction of radio-wave propagation characteristics in tunnel environments part 1 : Theory. IEEE Transactions on Antennas and Propagation, 46(9) :1328-1336, 1998. 